Ann. Biol. anim. Bioch. Biophys., I974, 14 (4-B), 837-844.

\title{
RÉPARTITION TISSULAIRE \\ DE LA RADIOACTIVITÉ CHEZ LE MOUTON AYANT REÇU DE LA ${ }^{75}$ SE-SÉLÉNOMÉTHIONINE ( $\left.{ }^{1}\right)$
}

\author{
M. HIDIROGLOU et K. J. JENKINS \\ Institut de Recherches zootechniques \\ (Animal Research Institute), \\ Ministère de l'A griculture, \\ Ollawa (Canada)
}

\section{RÉSUMÉ}

Par voie buccale ou intraveineuse, nous avons administré de la ${ }^{75}$ Se-sélénométhionine à 30 moutons, à raison de $5,5 \mu \mathrm{Ci}$ par $\mathrm{kg}$ de poids vif. Ces animaux ont été abattus 3,5 et 24 heures après l'administration de l'isotope. Dix moutons ont été utilisés aux intervalles de temps précités. Des radioactivités maximales ont été obtenues dans les tissus 24 heures après l'administration orale, 3 et 5 heures après l'administration intraveineuse (IV), ces dernières étant toujours plus hautes que les premières. C'est ainsi que dans certains tissus à forte synthèse protéique tel le pancréas par exemple, le marquage le plus élevé a été noté. Des radioactivités plasmatiques plus élevées que celles des érythrocytes ont été observées en général plutôt après une administration intra-veineuse qu'orale. Il y a lieu de mentionner que ces radioactivités étaient en majeure partie incorporées dans les protéines.

Les mesures de radioactivité effectuées sur le sang entier ont montré que durant les $3^{\circ}$ premières minutes suivant l'administration du radioisotope par voie intraveineuse, il y a eu une baisse subite suivie d'une remontée atteignant un pic de radioactivité à 60 minutes, puis à nouveau une décroissance graduelle et continue.

\section{INTRODUCTION}

La sélénométhionine joue un rôle important dans la nutrition en sélénium des ruminants, du fait que cet acide aminé prédomine proportionnellement aux autres formes séléniées des fourrages. (JENKINS et HidIROGLOU, I967). Ainsi, en analysant la luzerne, nous trouvons que plus de $70 \mathrm{p}$. Ioo de son contenu en sélénium se présente

(1) Contribution $n^{\circ} 540$ Animal Research Institute, Ottawa, Canada. 
sous forme de sélénométhionine (PETERSON et SPEDDING, I963). En outre, bien que l'emploi de la ${ }^{75}$ Se-sélénométhionine dans les scintigraphies de l'homme (SulLivan et al., I966) ait suscité un certain intétêt quant à son métabolisme chez les animaux de laboratoire (AwwAD et al., I967), très peu d'études ont été publiées à ce sujet chez les ruminants (HIDIROGLou et JENkins, I972). Le présent mémoire est consacré à la distribution de la radioactivité dans les tissus du mouton $24 \mathrm{~h}$, ou moins, après une administration de ${ }^{75}$ Se-sélénométhionine par les voies orale ou intraveineuse.

\section{MATÉRIEL ET MÉTHODES}

L'expérience a été effectuée sur 30 moutons de race Shropshire nourris pendant plusieurs mois avec un foin contenant 0,02-0,03 p.p.m. de sélénium.

Par voie buccale ou voie intraveineuse, de la ${ }^{75}$ Se-sélénométhionine (act. spécifique I $\mathrm{mCi} / \mathrm{mg}$ de sélénométhionine), a été administrée à ces 30 moutons à raison de $5.5 \mu \mathrm{Ci}$ par $\mathrm{kg}$ de poids vif. Les animaux ont été abattus 3,5 et $24 \mathrm{~h}$ (tabl. I) après l'administration de l'isotope, et chaque fois Io moutons ont été affectés aux intervalles de temps précités. Il y a lieu de préciser que les animaux de poids égal ont été utilisés par paire, l'une recevant le radioisotope par voie orale et l'autre par voie intraveineuse. Après l'administration de la ${ }^{75}$ Se-sélénométhionine, les animaux ont été placés dans des cages à métabolisme. Du sang a été recueilli aux intervalles de temps indiqués au tableau 2. Il a été centrifugé à I ooo g pendant I 5 minutes a fin de séparer le plasma des érythrocytes. Le plasma a par la suite subi les transformations ci-après : précipitation avec de l'acide trichloroacétique (ATA) 20 p. roo puis lavage du sédiment à l'ATA deux fois, à l'alcool à $80^{\circ}$ et ensuite à l'éther.

\section{Mesure de la radioactivité}

Des aliquots en triple (environ 50-100 mg) des différents tissus frais (tabl. I) ont été dissous dans $5 \mathrm{ml}$ d'hydroxide d'hyamine et leur radioactivité a été mesurée à l'aide d'un spectromètre Packard, Modèle 3002 . Il en a été de même de la radioactivité du plasma et des érythrocytes, laquelle a été aussi mesurée en triplicata (tabl. 2 et 3). En outre, la radioactivité du sang entier de six moutons a été mesurée 5 minutes après l'administration intraveineuse du radioisotope, puis toutes les ıo minutes au cours de la première heure (fig. I).

\section{Analyses statistiques}

Elles ont été conduites au moyen de l'analyse de variance. Étant donné que l'écart-type de la moyenne était proportionnel à la moyenne, il s'est avéré nécessaire de se servir de la transformation logarithmique afin de stabiliser la variance (SNEDECor et Cochran, I967). A la suite de ces transformations, les écarts-types furent plus normalement répartis autour de la moyenne.

\section{RÉSULTATS}

\section{A. - Radioactivités dans les tissus}

Si on compare les activités tissulaires en fonction du temps qui a suivi 1'administration orale de cet acide aminé sélénié, on remarque que les radioactivités sont plus faibles à 3 ou 5 heures qu'à 24 heures (tabl. I) temps ou le plus de radioactivité se trouve absorbée par le jéjunum du mouton (HIDIRogrou et JENkins, 1973). 
SÉLÉNOMÉTHIONINE CHEZ LE MOUTON

\begin{tabular}{|c|c|c|c|c|c|}
\hline 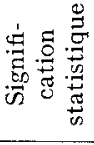 & * & $* *$ & * & & $*$ \\
\hline$\stackrel{\bullet}{\leftrightarrow}$ & $\begin{array}{l}5: \\
8 \\
0 \\
0\end{array}$ & $\frac{m}{s}=$ & $\begin{array}{l}5: 5 \\
0 \\
0\end{array}$ & 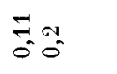 & $\begin{array}{l}\text { 范: } \\
8\end{array}$ \\
\hline 葓 & 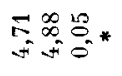 & 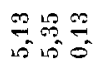 & 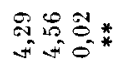 & $\begin{array}{l}8: 8 \\
8 \\
5\end{array}$ & 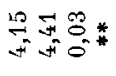 \\
\hline 20 & 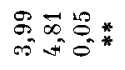 & 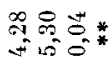 & 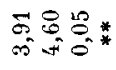 & $\begin{array}{l}\infty \\
\infty \\
\infty \\
\sin ^{-1}=0 \\
0\end{array}$ & $\begin{array}{l}\infty \\
\infty \\
\infty \\
\infty \\
\infty\end{array}$ \\
\hline$\infty$ & $=5$ & $\frac{8}{4}=\frac{9}{2}$ & 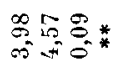 & 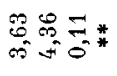 & 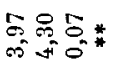 \\
\hline
\end{tabular}

$2 \infty$

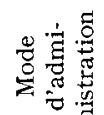

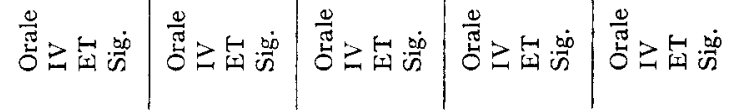

\begin{tabular}{|c|c|c|c|c|c|}
\hline 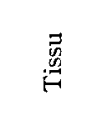 & 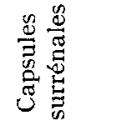 & 记㺃 & 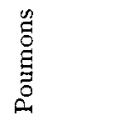 & 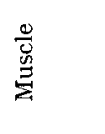 & $\begin{array}{l}\mathbf{3} \\
8\end{array}$ \\
\hline 莺 & $\stackrel{*}{*}$ & * & * & $*$ & \\
\hline 皇 & $\begin{array}{l}\infty \\
: 8 \\
0 \\
0\end{array}$ & $\begin{array}{l}80 \\
: 0 \\
0\end{array}$ & $\begin{array}{l}\mathscr{8}: 19 \\
\theta_{0}^{\circ}\end{array}$ & $\begin{array}{lll}\infty & 10 \\
0 & = \\
0 & 0\end{array}$ & \\
\hline$\sigma^{*}$ & 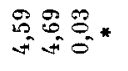 & 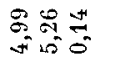 & 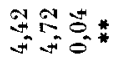 & 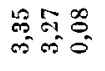 & \\
\hline 100 & 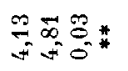 & 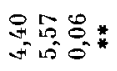 & 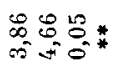 & 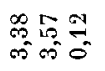 & \\
\hline
\end{tabular}

\begin{tabular}{|c|c|c|c|c|}
\hline ס & 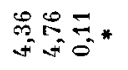 & 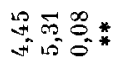 & 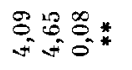 & 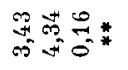 \\
\hline 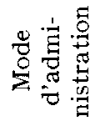 & 莺 & 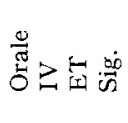 & 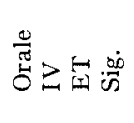 & 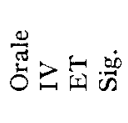 \\
\hline 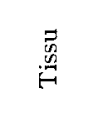 & 总 & 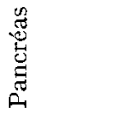 & 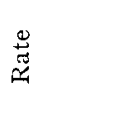 & $\frac{0}{\ddot{m}}$ \\
\hline
\end{tabular}


Par contre, lors de l'administration de la ${ }^{75}$ Se-sélénométhionine par voie IV. aucune différence significative n'a été observée $(P>0,05)$ dans la concentration de radioactivité tissulaire au cours des 3,5 et 24 heures.

En comparant à présent les activités tissulaires suivant les modes d'administration employés, on a remarqué qu'exception faite des radioactivités enregistrées à $24 \mathrm{~h}$ dans les reins, le pancréas, les muscles et la bile, et à $5 \mathrm{~h}$ dans les deux derniers tissus, les radioactivités tissulaires étaient toujours plus hautes après une administration intraveineuse plutôt qu'orale. De 3 à 5 heures après l'administration IV du radioisotope, on a observé que les concentrations de radioactivité pancréatique et rénale étaient nettement plus hautes que dans les autres tissus et avaient tendance à persister même lors de l'échantillonnage de $24 \mathrm{~h}$. De remarquables concentrations de radioactivité ont aussi été notées dans certains autres tissus tels que les capsules surrénales et le foie, suivies de celles de la rate et du poumon. Après une période de 3 et 5 heures, après l'administration orale, le pancréas se classait encore en tête, suivi de près par le foie, les reins et les capsules surrénales. Pendant ce temps, la concentration de radioactivité dans les différents tissus suivait plus ou moins le même ordre de classement que lors de l'administration IV. Au bout de 24 heures après l'administration, orale, la plus forte concentration s'observait dans les reins, puis dans le pancréas, les capsules surrénales et le foie.

\section{B. -- Radioactivité dans le plasma}

Si des radioactivités nettement plus élevées se sont manifestées dans le plasma au cours des deux premières heures qui ont suivi une administration intraveineuse de ${ }^{75}$ Se-sélénométhionine, ces différences se sont atténuées dans les heures suivantes tout en laissant cependant la priorité à une radioactivité légèrement plus haute chez les sujets administrés par voie intraveineuse (tabl. 2). Une heure après 1'administra-

TABLEAU 2

Radioactivité dans le plasma du mouton

après administration d'une seule dose de ${ }^{75}$ Se-sélénométhionine par voie orale ou intraveineuse

(Logarithmes de coups/mn/ml plasma)

\begin{tabular}{c|c|c|c|c}
\hline \hline $\begin{array}{c}\text { Heures après } \\
\text { administration }\end{array}$ & Orale & Intraveineuse & ET & Signification \\
& & & & \\
\hline & & & & \\
\hline 1 & 3,61 & 4,41 & 0,06 & $* *$ \\
2 & 4,02 & 4,37 & 0,04 & $* *$ \\
3 & 4,23 & 4,38 & 0,06 & \\
4 & 4,32 & 4,36 & 0,06 & \\
5 & 4,33 & 4,36 & 0,05 & \\
6 & 4,31 & 4,34 & 0,05 & \\
7 & 4,30 & 4,33 & 0,05 & \\
24 & 4,15 & 4,2 & 0,02 & \\
\hline
\end{tabular}

+ : Voir tableau 1 .

** $: \mathrm{P}<0,01$. 
tion IV de l'isotope, go p. Ioo de la radioactivité plasmatique se trouvait dans l'insoluble ATA (acide tricholoracétique à $20 \mathrm{p}$. IOO), et ce taux a continué d'augmenter pour atteindre $95 \mathrm{p}$. IOO à $6 \mathrm{~h}$ et $99 \mathrm{p}$. Ioo à $24 \mathrm{~h}$.

Une heure après l'administration orale, $80 \mathrm{p}$. Ioo de la radioactivité du plasma était incorporée dans les protéines, augmentant progressivement pour atteindre $90 \mathrm{p}$. IOo à $24 \mathrm{~h}$, ce qui confirme des observations antérieures (HIDIROGLOU et JENKINS, I972).

\section{C. - Radioactivité dans les érythrocytes}

La radioactivité des érythrocytes, comme celle du plasma, était toujours plus haute après une administration intraveineuse plutôt qu'orale, avec une différence significative $(P<O, O I)$ durant les deux premières heures. Pendant cette courte période d'échantillonnage, la radioactivité a été plus haute dans le plasma que dans les érythrocytes.

\section{TABLEAU 3}

Radioactivité dans les érythrocytes du mouton après administration d'une seule dose de ${ }^{\mathbf{7 5}}$ Se-sélénométhionine par voie orale ou intraveineuse (Logarithmes de coups/mn/ml plasma)

\begin{tabular}{|c|c|c|c|c|}
\hline $\begin{array}{c}\text { Heures après } \\
\text { administration }\end{array}$ & Orale & Intraveineuse & ET & Signification \\
\hline 1 & 2,90 & 3,53 & 0,06 & $* *$ \\
\hline 2 & 3,30 & 3,56 & 0,01 & $* *$ \\
\hline 3 & 3,43 & 3,56 & 0,05 & \\
\hline 4 & 3,47 & 3,54 & 0,06 & \\
\hline 5 & 3,52 & 3,50 & 0,07 & \\
\hline 6 & 3,50 & 3,52 & 0,06 & \\
\hline 7 & 3,50 & 3,51 & 0,06 & \\
\hline 24 & 3,47 & 3,44 & 0,05 & \\
\hline $\begin{array}{l}+: \text { Voir } \\
* *: \mathrm{P}<\end{array}$ & au 1. & . & & \\
\hline
\end{tabular}

\section{D. - Radioactivité dans le sang entier}

En ce qui concerne les radioactivités du sang entier mesurées aux courts intervalles de la $\mathrm{I}^{\text {re }}$ heure qui a suivi l'administration $I V$, on a remarqué (fig. I), qu'il y a eu une baisse continuelle de celles-ci au cours des 30 premières minutes. C'est ainsi qu'à ce moment-là, la radioactivité était seulement 44 p. Ioo de celle mesurée à 5 minutes. Par la suite on a constaté une remontée et à 60 minutes, la radioactivité atteignait 78 p. Ioo de celle indiquée à 5 minutes. Les mesures suivantes ont accusé une baisse continuelle. 


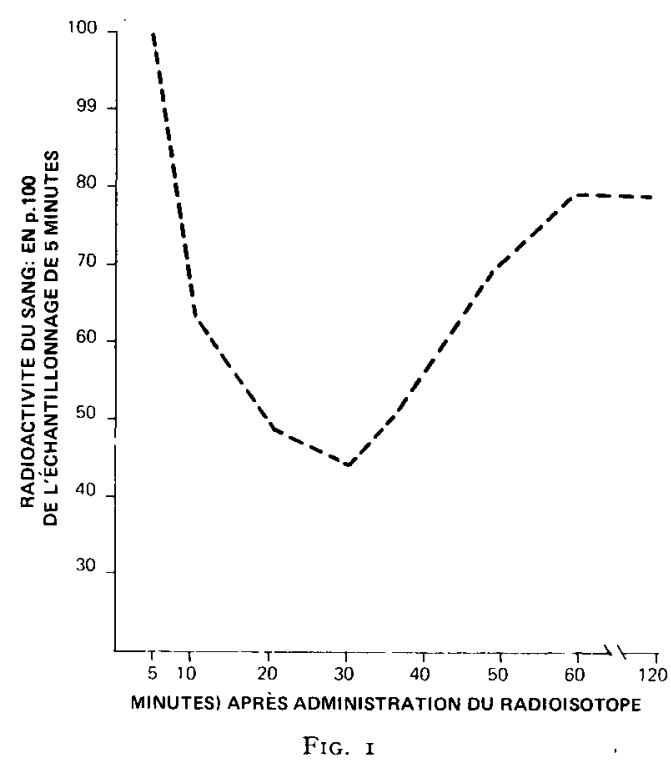

\section{DISCUSSION}

Les résultats que nous venons de présenter démontrent que les activités spécifiques les plus hautes sont trouvées dans les pancréas, reins et foie du mouton ce qui confirme le travail de Hanson et Jacobson (I966) chez la Souris, et celui de AwWAD (I966) chez le Rat. Ces lieux de prédilection sont, selon VANLERENBERGHE et coll. (I968) dus au fait que cet acide aminé s'incorpore très rapidement dans les protéines de ces trois organes. Le fait que 3 heures après une administration IV, les concentrations les plus hautes parmi les tissus échantillonnés aient été trouvées dans le pancréas du mouton confirme les résultats de BLAU et MANSke (I96I). D'après ces auteurs, cet organe est le lieu de prédilection pour cet acide aminé sélénié du fait que 7 p. Ioo de la sélénométhionine y a été concentrée $\mathrm{I}$ à 2 heures après son administration par voie intraveineuse. Indépendemment du mode d'administration, le taux de marquage des muscles se rangeait parmi les plus faibles, ce qui a déjà été rapporté par NEEThiting et al. (I968), Hidirogiou et Jenkins (1972). Une concentration élevée de la radioactivité a été remarquée dans la bile 3 heures après une injection intraveineuse, avec une décroissance rapide à 5 et 24 heures, ce qui semble confirmer les résultats de VANLERENBERGHE et coll. (I968) chez le Rat.

Nos résultats sur la nature de produits marqués présents dans le plasma concordent avec ceux rapportés par EVATT et LEVIN (Ig6I) sur les lapins. Ces auteurs ont également trouvé que plus de $99 \mathrm{p}$. Ioo de la radioactivité plasmatique était incorporée dans les protéines plasmatiques 24 heures après une administration IV de ${ }^{75}$ Se-sélénométhionine.

En ce qui concerne la différence du taux de l'incorporation dans les plasmas et les érythrocytes nos résultats semblent confirmer ceux de ROSENFELD et EPPSON 
(1964) qui, lors de l'administration de ${ }^{75}$ Se-sélénite, ont trouvé plus de radioactivité dans le plasma que dans les érythrocytes du mouton dans les premières heures.

Nos résultats sur l'évolution des radioactivités du sang entier semblent confirmer ceux obtenus chez le Rat par OLDENDORF et KITANo (I963). En effet, d'après ces auteurs, il se peut que la ${ }^{75}$ Se-sélénométhionine administrée par voie intraveineuse au rat soit transférée au foie où elle se trouve incorporée dans les protéines pour être ensuite retransférée dans le sang.

Il y a cependant lieu de mentionner que, d'après STERNBERG et Imbach (1967), le foie n'est pas indispensable pour l'incorporation de la sélénométhionine dans les protéines plasmatiques. Selon ces auteurs, il se peut que cet acide aminé soit intégré dans les protéines plasmatiques par transpeptidation lors de sa circulation dans le courant sanguin. Mais il est évident que ceci reste à prouver, car rien n'exclut la possibilité d'un transfert de ${ }^{75}$ Se seul, sans son radical, dans une autre molécule.

\section{CONCLUSION}

L'étude de la répartition de la radioactivité au cours des 24 heures suivant l'administration orale ou IV donne une indication sur le lieu de prédilection de cet acide aminé sélénié dont les radioactivités particulièrement élevées ont été enregistrées dans le pancréas, reins et foie du mouton. En outre, un fait remarquable qui a été souligné est les rapides changements de concentrations de la radioactivité dans le sang au cours des premières minutes qui suivent l'administration IV de la ${ }^{75} \mathrm{Se}$ sélénométhionine, ce qui donne une indication quant à la vitesse de son métabolisme.

Recu pour publication en juillet 1974.

\section{SUMMARY}

\section{DISTRIBUTION OF RADIOACTIVITY \\ IN THE TISSUES OF SHEEP GIVEN ${ }^{75}$ SE-SELENOMETHIONINE}

We gave oral and intravenous doses of ${ }^{75}$ Se-selenomethionine to 30 sheep in the proportion of $5.5 \mu \mathrm{Ci}$ per $\mathrm{kg}$ of liveweight. These animals were slaughtered at 3.5 and 24 hours after isotope administration. Ten sheep were used at each of the intervals mentioned. Maximum radiactivity was obtained in the tissues 24 hours after oral administration, and 3 and 5 hours after intravenous (IV), the latter doses being stronger than the former. Highest labeling was observed in some tissues having high protein synthesis, such as the pancreas. Plasma radioactivity, higher than that of the erythrocytes, was noted more often after IV than after oral administration. Most of this radioactivity was incorporated in proteins.

Radioactivity measurements of whole blood showed that during the first 30 minutes following intravenous injection of the isotope, there is a sudden drop, then a rise reaching a peak of radioactivity at 60 minutes, followed again by a gradual and continuous decrease.

\section{RÉFÉRENCES BIBLIOGRAPHIQUES}

Awwad K. J., Adelstern S. J., Potchen E. J., Dealy J. B. Jr., I967. The interconversion and reutilization of injected ${ }^{75}$ Se-selenomethione in the rat. J. Biol. Chem., 242, 492-500.

Awwad K. J., Potchen E. J., Adelstein S. J., Dealy J. B., I966. The regional distribution of ${ }^{75}$ Se-selenomethionine in the rat. Metabolism Clin. Expt., 15, 370-378.

Annales de Biologie animale. - r974. 
Blau M., Manske R. F., I96I. The pancreas specificity of ${ }^{75}$ Se-selenomethionine. J. Nucl. Med. 2, $102-105$.

Evatt B., Levin J., I969. Measurement of Thrombopoiesis in rabbits using ${ }^{75}$ Se-selenomethionine Clin. Invest., 48, 1615-1626.

Hanson Eskil, Jaconson S. O., 1966 . Uptake of ${ }^{75}$ Se-selenomethionine in the tissues of the mouse studied by whole-body autoradiography. Biochem. Biophys. Act., 115, 285-293.

Hidiroglou M., Jenkins K. J., I972. Le sort du radiosélénium administré dans le rumen ou dans la caillette du mouton. Ann. Biol. Anim. Bioch. Biophys., 12, 599-616.

Jenkins K., Hidiroglou M., 1967 . The incorporation of ${ }^{75}$ Se-selenite into dystrophogenic pasture grass. The chemical nature of the selenocompounds formed and their availability to young ovine. Can. J. Biochem., 45, ro27-ro4o.

Neethling L. P., Brown J. M. M., Dewet P. J., rg68. The toxicology and metabolic rate of selenium in sheep. J.S. Afr. Vet. Med. Ass., 39 (3), 25-33.

OldendorF H. W., Kitano M., I963. Selenomethionine reappearance in blood following intravenous injection. J. Nuc. Med., 4, $23 \mathrm{I}-233$.

Peterson P. J., Spedding D. J., I963. The excretion by sheep of ${ }^{75}$ Se incorporated into red clover (Trifolium mature L.). The chemical mature of the excreted selenium and its uptake by three plant species. N. Z. J. Agr. Res., 6, 13-23.

Rosefeld I., Eppson H. F., r964. Metabolism of selenium in sheep, p. 53-64. In " Metabolic effects and metabolism of Se in animals ». Agric. Exp. Station, Wyoming., Bulletin 4I4.

SNedecor G. W., Cochran W. G., I967. Statistical methods. Sixth ed. p. 239 Iowa State University.

SternberG J., IмвасH A., I967. Metabolic studies with seleniated compounds. II. Turnover studies with ${ }^{75}$ Se-Methionine in rats. J. Applied Rad. Isotop., 18, 569-578.

Sullivan B. H., Meaney T. F., Rodriguez-Antunez A., Brown C. H., I966. Diagnosis of carcinoma of the pancreas by selective coeliac arteriography and ${ }^{75}$ Se-photoscanning. Recent advances in gastro-enterology, 4, 382-384.

Vanlerenberghe J., Turpin N., Cassaigne R., I968. Distribution régionale et excrétion biliaire de Sélénométhionine chez le Rat. C. R. Acad. Sc. Paris, 267, 100-102. 